\title{
Mit wissen kompakt das neue Jahr aktiv beginnen
}

Die hohe Inzidenz von Schlafproblemen lässt erahnen wie ubiquitär dieses Problem in der Gesellschaft ist und dennoch fristet das interdisziplinäre Thema „Schlafmedizin“ ein Schattendasein. Im Netzwerk der interdisziplinären Schlafmedizin übernimmt die Zahnmedizin eine aktive Schlüsselposition, so spielt sie z.B. bereits in der Diagnostik eine zentrale Rolle.

Aus diesem Grund widmet sich die im Februar erscheinende Ausgabe wissen kompakt dem wichtigen interdisziplinären Thema Schlafmedizin. Neuestes zum Thema liegt dann für Sie bereit, um gut informiert und aktiv in das neue Jahr zu starten:

"Schlafbezogene Atmungsstörungen"

Prof. Dr. Winfried J. Randerath und Dr. Sebastian Böing

„Die Unterkieferprotrusionsschiene im

interdisziplinären Kontext"

Prof. Dr. Dr. Elmar Esser, Axel Röers, Dr. Laura Schenk
"Obstruktive Schlafapnoe und Schnarchen"

Prof. Dr. Dr. Hans Pistner

Die Teilnahme an den Continuing-Medical-Education (CME)Fortbildungen erfolgt online auf www.springerzahnmedizin.de und ist für die Mitglieder des Freien Verbandes Deutscher Zahnärzte (FVDZ) sowie für Abonnenten von wissen kompakt kostenlos. Beides trifft nicht auf Sie zu? Die entsprechenden Anträge finden Sie in dieser Ausgabe auf Seite 45/46.

wissen kompakt - gebündelte zertifizierte zahnärztliche Fortbildung

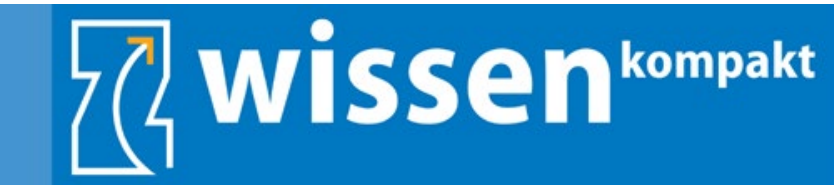

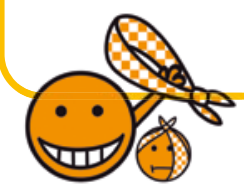 \\ Bestellcoupon

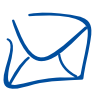

Der neue Logk mit dem \section{モ gewissen Lächeln.}

Stck. Größe S

Stck. Größe S

zu je Euro 20,95*

Stck. Größe S

Stck. Größe S

Stck. Größe S
Stck. Größe S
T-Shirts

\section{weiß}

blau

bordeaux

Polohemden

weiß

blau

Stck. Größe XS

bordeaux

Stck. Größe XS

Bitte faxen oder schicken an:

\section{8 / 8551132}

Dynadent

Service für Zahnärzte GmbH

Mallwitzstraße 16

zu je Euro 17,95* | Ab 5 Stück zu je 15,95* pro Stück

Stck. Größe M
Stck. Größe M
Stck. Größe M

Stck. Größe M

Ab 5 Stück zu je 18,95* pro Stück

Stck. Größe M
Stck. Größe M
Stck. Größe M

$\begin{array}{rr}\text { Stck. Größe L } & \text { Stck. Größe XL } \\ \text { Stck. Größe L } & \text { Stck. Größe XL } \\ \text { Stck. Größe L } & \text { Stck. Größe XL } \\ \text { pro Stück } & \\ \text { Stck. Größe L } & \text { Stck. Größe XL } \\ \text { Stck. Größe L } & \text { Stck. Größe XL } \\ \text { Stck. Größe L } & \text { Stck. Größe XL } \\ & \text { *alle Preise inkl. MwSt. und Versand }\end{array}$

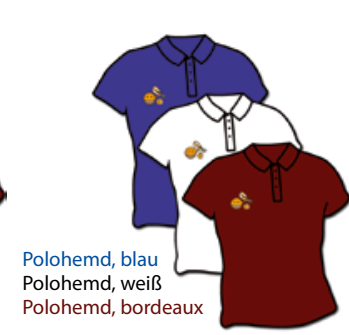

$-V D=\frac{19}{20}$

\section{S}

Dynadent GmbH

\begin{tabular}{l} 
Name \\
\hline Straße \\
\hline PLZ/Ort \\
\hline Datum, Unterschrift
\end{tabular}

